\title{
Variability in Antithrombotic Therapy Regimens Peri-TAVR: A Single Academic Center Experience
}

Jeffrey E. Rossi - Andrew Noll - Brian Bergmark - James M. McCabe ·

David Nemer - David R. Okada · Anant Vasudevan · Michael Davidson ·

Frederick Welt • Andrew Eisenhauer · Pinak Shah •

Robert Giugliano

To view enhanced content go to www.cardiologytherapy-open.com

Received: August 21, 2015 / Published online: September 23, 2015

(c) The Author(s) 2015. This article is published with open access at Springerlink.com

\section{ABSTRACT}

Introduction: The aim of this study was to describe peri-procedural antithrombotic use in patients undergoing transcatheter aortic valve replacement (TAVR) at a single academic medical center.

Methods: Retrospective collection of antiplatelet and anticoagulant use during the index hospitalization for all patients undergoing TAVR at our institution from April 2009 through March 2014.

M. Davidson: deceased January 20, 2015.

Electronic supplementary material The online version of this article (doi:10.1007/s40119-015-0050-2) contains supplementary material, which is available to authorized users.

J. E. Rossi $(\bowtie)$ - A. Noll · B. Bergmark · D. Nemer . D. R. Okada · A. Vasudevan · M. Davidson .

A. Eisenhauer · P. Shah · R. Giugliano Division of Cardiovascular Medicine, Brigham and Women's Hospital, Boston, MA, USA

e-mail: jerossi27@gmail.com

J. M. McCabe

Division of Cardiology, University of Washington,

Seattle, WA, USA

F. Welt

Division of Cardiovascular Medicine, University of Utah, Salt Lake City, UT, USA
Results: Of a total of 255 patients undergoing the procedure, 132 (51\%) had an indication for anticoagulation pre-TAVR and 92 (70\% of those with an indication) were on treatment. On discharge, 106 patients (44\% of total surviving to discharge, $73 \%$ of those surviving with an indication for anticoagulation) were treated with oral anticoagulation. Of these patients, 89 (84\%) were discharged on aspirin and an oral anticoagulant without clopidogrel. Only 122 (51\% of total patients) were discharged on the regimen of aspirin and clopidogrel alone.

Conclusion: Peri-procedural antithrombotic regimens vary greatly following TAVR. More than half of patients have an indication for anticoagulation following the procedure. Most patients at our institution who require anticoagulation are discharged on aspirin and an oral anticoagulant, though the optimal regimen requires further investigation.

Keywords: Anticoagulation; Antithrombotic therapy; Atrial fibrillation; Dual antiplatelet therapy (DAPT); Stroke; Structural heart disease; Transcatheter aortic valve replacement (TAVR); Transfemoral aortic valve implantation (TAVI); Triple therapy 


\section{INTRODUCTION}

Current guidelines call for lifelong aspirin (acetylsalicylic acid; ASA) with consideration of up to 6 months of clopidogrel following transcatheter aortic valve replacement (TAVR) [1]. Recently, small studies have questioned the necessity of dual antiplatelet therapy (DAPT) following the procedure [2]. Furthermore, many patients receiving TAVR have a pre-existing indication for oral anticoagulation (OAC), most commonly atrial fibrillation (AF) [3]. To date, there have been little data formally addressing the optimal combination of antiplatelet and anticoagulant medications after TAVR, particularly in patients with an indication for OAC.

A 2012 expert consensus document supported by the American College of Cardiology (ACC) and Society of Thoracic Surgeons (STS) suggests treating these patients with ASA and an anticoagulant, omitting clopidogrel [4]. The 2014 ACC/American Heart Association (AHA) valvular disease guidelines give a IIb recommendation for 6 months of treatment with ASA and clopidogrel after TAVR, but do not comment on patients with an indication for anticoagulation [1]. Current European Society of Cardiology (ESC) guidelines state that in TAVR patients with AF, a single antiplatelet combined with an anticoagulant is generally used but treatment decisions should be made based on the perceived bleeding risk of an individual patient [5]. Actual treatment patterns in clinical practice are unknown and are likely to vary by physician and institution [6]. Therefore, we assessed the current practice of antithrombotic treatment following TAVR at a single, large academic center.

\section{METHODS}

We performed manual chart review of all patients undergoing TAVR from April 2009 through March 2014 at Brigham and Women's Hospital in Boston, MA, USA. Pre-TAVR antithrombotic regimens were obtained from admission medication reconciliations or prior clinic notes when possible. Post-procedural medications were obtained from the hospital's electronic medication administration log and patient discharge summaries. Additional data were collected using the electronic medical record. Events were classified according to Valve Academic Research Consortium (VARC) definitions [7]. All procedures followed were in accordance with the ethical standards of the responsible committee on human experimentation (institutional and national) and with the Helsinki Declaration of 1964, as revised in 2013. Informed consent was obtained from all patients for being included in the study.

\section{RESULTS}

Of 255 total patients (mean age 81 years, 48\% female), 169 (66\%) had transfemoral, 44 (17\%) had transapical, and 42 (16\%) had an alternative access site TAVR (Table 1). One hundred and thirty-one (51\%) patients had an indication for OAC pre-TAVR, of which 122 (48\%) had $\mathrm{AF}$ and $\mathrm{CHADS}_{2}$ (congestive heart failure, hypertension, age, diabetes, stroke) score $>1,6$ (2\%) had a history of deep vein thrombosis/pulmonary embolism, and 3 (1\%) had other indications. Of patients with an indication for OAC, 92 (70\%) were on OAC prior to the procedure, the majority $(88 \%)$ of whom were treated with warfarin. Twenty-nine (11\%) of the total cohort were on DAPT prior to 
Table 1 Baseline characteristics

\begin{tabular}{ll}
\hline Characteristic & Value \\
\hline Age, years & $80.6 \pm 9.77$ \\
BMI, km/m ${ }^{2}$ & $27.3 \pm 6.66$ \\
White & 94.9 \\
Male & 51.8 \\
Hypertension & 90.2 \\
Diabetes & 39.6 \\
Atrial fibrillation or flutter & 48.2 \\
Permanent & 38.2 \\
Paroxysmal & 45.5 \\
n/a & 16.3 \\
CHADS 2 score & $3.29 \pm 1.05$ \\
NYHA class III/IV & 93 \\
eGFR, mL/min/1.73 m ${ }^{2}$ & $57.8 \pm 29.5$ \\
Dialysis & 3.1 \\
MI & 21.6 \\
PCI & 29.0 \\
CABG & 36.1 \\
Peripheral arterial disease & 22.0 \\
Cerebrovascular disease & 13.3 \\
Chronic lung disease & 40.8 \\
DVT/PE & 9.0 \\
GI Bleeding & 14.9 \\
Hemoglobin, g/dL & $11.1 \pm 1.78$ \\
Platelets, k/ $\mu \mathrm{L}$ & $201.7 \pm 82.6$ \\
LVEF, \% & $53.8 \pm 14.68$ \\
Aortic valve area, cm ${ }^{2}$ & $0.66 \pm 0.17$ \\
Aortic valve peak velocity, m/sec & $4.29 \pm 0.63$ \\
Moderate-Severe MR & 39.3 \\
\hline & \\
Transfemoral & \\
\hline
\end{tabular}

Table 1 continued

\begin{tabular}{lc}
\hline Characteristic & Value \\
\hline Other valve access site & 16.4 \\
\hline Values are mean \pm standard deviation or percentage \\
$B M I$ body mass index, $C A B G$ coronary artery bypass \\
grafting, $C H A D S_{2}$ congestive heart failure, hypertension, \\
age, diabetes, stroke, $D V T / P E$ deep vein thrombosis/ \\
pulmonary embolism, eGFR estimated glomerular \\
filtration rate, $G I$ gastrointestinal, $L V E F$ left ventricular \\
ejection fraction, $M I$ myocardial infarction, $M R$ mitral \\
regurgitation, $n / a$ not available, $N Y H A$ New York Heart \\
Association, $P C I$ percutaneous coronary intervention
\end{tabular}

the procedure (most for recent coronary stenting) and 5 (2\%) were receiving triple therapy with two antiplatelet agents and an anticoagulant. Complete baseline and discharge antithrombotic use is shown in Table 2.

All patients had OAC held prior to the procedure and only 12 (13\%) patients on baseline OAC were bridged with a parental anticoagulant prior to TAVR. There were 155 patients (65\%) who had an indication for OAC post-TAVR (including 19 with new onset AF), and of these 77 (50\%) were bridged with parental anticoagulation after the procedure. Of the 145 patients with an indication for OAC who survived the initial hospitalization, 106 (73\%) were discharged on an antithrombotic regimen that included an anticoagulant. Of the 16 surviving patients with new onset AF, 11 (69\%) were newly started on OAC after TAVR.

In the total cohort there were 15 deaths, 13 strokes (10 ischemic, 2 hemorrhagic, and 1 unspecified) and 29 major bleeding events prior to discharge. The median time to stroke was 2 days post-procedure. Seven out of 13 patients with in-hospital stroke (5 ischemic) had longstanding AF. Of the 5 patients with 
Table 2 Admission and discharge antithrombotic regimens in patients undergoing transcatheter aortic valve replacement

\begin{tabular}{lccccccc}
\hline Admission antithrombotic regimen & \multicolumn{7}{l}{ Discharge antithrombotic regimen } \\
\cline { 2 - 8 } & SAPT $^{\mathbf{a}}$ & DAPT $^{\mathbf{b}}$ & OAC & SAPT + OAC & Triple $^{\mathbf{c}}$ & Died $^{\text {Total }^{2}}$ \\
\hline None & 4 & 16 & 0 & 4 & 1 & 5 & 30 \\
SAPT & 7 & 78 & 0 & 16 & 0 & 3 & 104 \\
DAPT & 1 & 21 & 0 & 4 & 1 & 2 & 29 \\
OAC & 0 & 1 & 2 & 21 & 7 & 2 & 33 \\
SAPT + OAC & 0 & 5 & 0 & 43 & 3 & 3 & 54 \\
Triple & 0 & 1 & 0 & 1 & 3 & 0 & 5 \\
Total & 12 & 122 & 2 & 89 & 15 & 15 & 255 \\
\hline
\end{tabular}

$A S A$ aspirin (acetylsalicylic acid), DAPT dual antiplatelet therapy, $O A C$ oral anticoagulation, $S A P T$ single antiplatelet therapy

${ }^{a} \mathrm{SAPT}=\mathrm{ASA}$ or $\mathrm{P} 2 \mathrm{Y} 12$ antagonist

${ }^{\mathrm{b}} \mathrm{DAPT}=\mathrm{ASA}+\mathrm{P} 2 \mathrm{Y} 12$ antagonist

${ }^{c}$ Triple $=\mathrm{ASA}+\mathrm{P} 2 \mathrm{Y} 12$ antagonist $+\mathrm{OAC}$

ischemic stroke, three were not on baseline OAC and two had OAC held without bridging prior to the procedure. Of 106 patients discharged on OAC, 89 (84\%) were treated with ASA + OAC. The most common discharge regimen for the 95 surviving patients without an indication for anticoagulation was DAPT (93\%) with only 7 (7\%) receiving ASA or clopidogrel alone.

\section{Limitations}

Our study was limited by retrospective collection of patient's antithrombotic regimens. In addition, the small number of events and lack of long-term follow-up precluded our ability to relate treatment medications with outcomes.

\section{CONCLUSIONS}

In conclusion, at our institution, post-procedural antithrombotic regimens in patients receiving TAVR are highly variable. Nearly two-thirds of patients had an indication for OAC post-TAVR. For patients with an indication for anticoagulation, treating physicians tended (84\%) to follow consensus guidelines that suggest a regimen of ASA + OAC following the procedure. Overall, DAPT remains the most frequent antithrombotic regimen at discharge, although $7 \%$ of patients were treated with a single antiplatelet agent alone. An analysis of the German Aortic Valve Registry (GARY) found similar results with $66 \%$ of patients discharged on DAPT and $27 \%$ discharged on an anticoagulant [8]. This study showed a higher rate of triple therapy with $16 \%$ of total patients discharged on DAPT plus an anticoagulant [8]. Whether these regimens truly represent the optimal balance between bleeding and thrombosis following TAVR, particularly in patients at high risk for adverse events like those with $\mathrm{AF}$, should be evaluated prospectively in future randomized trials. 


\section{ACKNOWLEDGMENTS}

No funding or sponsorship was received for this study or publication of this article. All named authors meet the International Committee of Medical Journal Editors (ICMJE) criteria for authorship for this manuscript, take responsibility for the integrity of the work as a whole, and have given final approval to the version to be published.

Disclosures James M. McCabe has received consultant fees/honoraria from Edwards, and is on the speaker's bureau for Abbott. Frederick Welt has received consultant fees/honoraria from Medtronic Inc. and Volcano Corporation, and is on the speaker's bureau for St. Jude Medical. Pinak Shah has received consultant fees/honoraria from Boston Scientific and St. Jude Medical. Robert Giugliano has received consultant fees/honoraria from Amgen, AngelMed, Daiichi Sankyo, GlaxoSmithKline, Johnson \& Johnson, Lexicon, Merck, Pfizer, Portola, and St. Jude Medical, and has participated in research for/received research grants from Amgen, Daiichi Sankyo, and Merck. Jeffrey E. Rossi, Andrew Noll, Brian Bergmark, David Nemer, David R. Okada, Anant Vasudevan, Michael Davidson, and Andrew Eisenhauer have nothing to disclose.

\section{Compliance with ethics guidelines All} procedures followed were in accordance with the ethical standards of the responsible committee on human experimentation (institutional and national) and with the Helsinki Declaration of 1964, as revised in 2013. Informed consent was obtained from all patients for being included in the study.

Open Access This article is distributed under the terms of the Creative Commons Attribution-
NonCommercial 4.0 International License (http://creativecommons.org/licenses/by-nc/4. $0 /$ ), which permits any noncommercial use, distribution, and reproduction in any medium, provided you give appropriate credit to the original author(s) and the source, provide a link to the Creative Commons license, and indicate if changes were made.

\section{REFERENCES}

1. Nishimura RA, Otto CM, Bonow RO, et al. 2014 AHA/ ACC guideline for the management of patients with valvular heart disease: a report of the American College of Cardiology/American Heart Association Task Force on Practice Guidelines. J Am Coll Cardiol. 2014;63(22):e57-185.

2. Ussia GP, Scarabelli $M$, Mule $M$, et al. Dual antiplatelet therapy versus aspirin alone in patients undergoing transcatheter aortic valve implantation. Am J Cardiol. 2011;108(12):1772-6.

3. Leon MB, Smith CR, Mack M, et al. Transcatheter aortic-valve implantation for aortic stenosis in patients who cannot undergo surgery. N Engl J Med. 2010;363(17):1597-607.

4. Holmes DR Jr, Mack MJ, Kaul S, et al. ACCF/AATS/ SCAI/STS expert consensus document on transcatheter aortic valve replacement. J Am Coll Cardiol. 2012;59(13):1200-54.

5. Joint Task Force on the Management of Valvular Heart Disease of the European Society of C, European Association for Cardio-Thoracic S, Vahanian A, et al. Guidelines on the management of valvular heart disease (version 2012). Eur Heart J. 2012;33(19):2451-2496.

6. Nijenhuis VJ, Stella PR, Baan J, et al. Antithrombotic therapy in patients undergoing TAVI: an overview of Dutch hospitals. Neth Heart J. 2014;22(2):64-9.

7. Leon MB, Piazza N, Nikolsky E, et al. Standardized endpoint definitions for transcatheter aortic valve implantation clinical trials: a consensus report from the Valve Academic Research Consortium. J Am Coll Cardiol. 2011;57(3):253-69.

8. Zeymer U, Zahn R, Gerckens U, Richard G, Figulla HR, Senges J. Antithrombotic therapy after transfemoral aortic valve implantation (TAVI). Potential hazard of triple-therapy. Eur Heart J. 322011:900. 